\title{
Infartos viscerales múltiples detectados por TAC en la aspergilosis invasiva
}

\author{
E. VÁZQUEZ MUÑOZ, M. MORADO ARIAS, J. A. SOBRINO DAZA \\ Servicios de Hematología y Cardiología . Hospital Universitario La Paz. Madrid
} MULTIVISCERAL INFARCTS ARE COMPLICATION OF INVASIVE
ASPERGILLOSIS

\begin{abstract}
RESUMEN
La aspergilosis es una infección causada por el hongo Aspergillus fumigatus. En la aspergilosis invasiva y diseminada se produce un cuadro muy grave caracterizado por fiebre, síndrome respiratorio y generalmen te muerte. Estas formas aparecen en la inmunosupresión severa. Pero hay además una forma grave de aspergilosis, la aspergilosis aórtica, que se presenta en enfermos que, sin defecto inmunológico alguno, han sido sometidos a reemplazamiento valvular o cirugía coronaria.

Damos a conocer dos casos de aspergilosis invasiva que cursaron con infartos viscerales múltiples: hepático, esplénico, renales, pancreáti$\cos$, tiroideos y cerebrales. Estos infartos fueron confirmados en la autopsia y parecían producidos por la ingente cantidad de hifas intravasculares, observadas en las áreas infárticas, que pudieron actuar a modo de émbolos sépticos, sin descartar una vasculitis aspergilar.

Pensamos que los infartos multiviscerales constituyen una complicación subestimada en la aspergilosis invasiva. Es necesario que aprendamos a reconocer estas formas emergentes de aspergilosis invasiva, que serán más frecuentes cada día por el aumento de enfermos inmunodeprimidos.
\end{abstract}

PALABRAS CLAVE: Aspergilosis invasiva. Infartos viscerales. Arteritis por Aspergillus. Hifas.

\begin{abstract}
Aspergillus fumigatus is one of the species of Aspergillus that causes aspergillosis. The clinical picture of invasive-disseminated aspergillosis is generally charactericed by fever and respiratory distress that usually follows a fatal course. This form of invesive aspergillosis is suffered by severely immunossupressed patients. There is another clinical form of severe aspergillosis, aortic aspergillosis, that appears after cardiac sur gery.

We present two cases of invasive aspergillosis complicated by multi ple visceral infarcts involving the liver, spleen, kidneys, pancreas, thi roid and brain. Infarcts were confirmed in necropsy and appeared to be caused by an overwhelming amount of intravascular hiphae, wich were observed in the infarcted areas acting forming septic embolus.

We believe that multi-visceral infarcts are an underestimed complication of invasive aspergillosis. Given the progressive increse in the population of immunossupressed patients, clinicians have to aware of all the possible presentations of invasive aspergillosis.
\end{abstract}

KEY WORDS: Invasive aspergillosis. Visceral infarcts. Arteritis por Aspergillus. Hiphae.

Vázquez Muñoz E, Morado Arias M, Sobrino Daza JA. Infartos viscerales múltiples detectados por TAC en la aspergilosis invasiva. An Med Interna (Madrid) 2002; 19: 530-532.

\section{INTRODUCCIÓN}

La aspergilosis en una infección causada por hongos micelares, muy ubicuos, que dan origen a formas clínicas diversificadas, moduladas por la inmunidad del huésped (1), entre las que destacan la aspergilosis broncopulmonar alérgica, el aspergiloma y la aspergilosis invasiva pulmonar. El último cuadro es altamente letal y cursa con fiebre, junto a grave afectación del estado general (2). Hay también otra forma de aspergilosis, la aortitis por Aspergillus, que produce un cuadro de émbolos sépticos generalizados, y cuya etiología parece que guarda relación con la circulación extracorpórea, prac- ticada en el reemplazamiento aórtico y en la cirugía coronaria $(3,4)$.

Las formas invasivas y diseminadas eran una rareza en el pasado por lo que siempre eran diagnosticadas en la necropsia, pero en el último tiempo han aumentado considerablemente su incidencia, como consecuencia del aumento de pacientes severamente inmudeprimidos. No es, pues, extraño que sus manifestaciones clínicas sean todavía poco conocidas. Por ello damos a conocer dos casos de aspergilosis generalizada que cursaron con múltiples infartos viscerales (cerebro, hígado, bazo y riñón, suprarrenales, tiroides, intestino y arteria cubital), en los que el estudio autópsico reveló una emboli-

Trabajo aceptado: 10 de abril de 2001

Correspondencia: E. Vázquez Muñoz. C/ La Campana nº 10. Ciudad Santo Domingo. 28120 Madrid 
zación micelar. Aunque se han descrito en la aspergilosis infartos viscerales, un cuadro multinfártico como el que presentaban nuestros dos pacientes es muy infrecuente.

\section{CASOS APORTADOS}

Caso 1: Paciente de 70 años, con adenopatías laterocervicales, submaxilares, axilares y mediastínicas. Estudio analítico: V de $\mathrm{S} 85$ $\mathrm{mm} / \mathrm{h}$; $\mathrm{Hct}^{\circ} 26 ; \mathrm{Hb} 9 \mathrm{~g} / \mathrm{dl}$; hematíes $3,1 \times 10^{6} \mathrm{~mm}$; leucocitos $2,8 \mathrm{x}$ $103 \mathrm{m \mu}$; ácido úrico 9,9 mg/dl . Resto de parámetros convencionales normales. Biopsia de médula ósea: Infiltrada por células de estirpe linfoide. Biopsia ganglionar: ganglio de arquitectura borrada, por proliferación de células de aspecto linfoide, con moderada cantidad de citoplasma y núcleos de contorno irregular; el aspecto histológico era compatible con un linfoma no Hodgkin de alto grado de malignidad. Se instauró un tratamiento quimioterápico con ciclofosfamida, vincristina y prednisona, con lo que se consiguió una desaparición de las adenopatías.

Al cabo de tres semanas se inicia fiebre, disnea aguda y tos. En la placa de torax se observaron infiltrados pulmonares, confirmados por TAC, que sugirieron entre otras posibilidades, una aspergilosis pulmonar invasiva. Dos días después apareció un síndrome neuroló gico con desorientación y descenso del nivel de conciencia, por lo que se practicó un TAC cerebral en el que se encontraron lesiones sugestivas de infartos corticales. El TAC abdominal reveló imágenes hipodensas, triangulares, compatibles con infartos isquémicos en el bazo y en el hígado (Fig. 1). Había asimismo infartos en el riñón (Fig. 2). Se instauró tratamiento con antibióticos y anfotericina B, pese a lo cual el paciente falleció al tercer día.

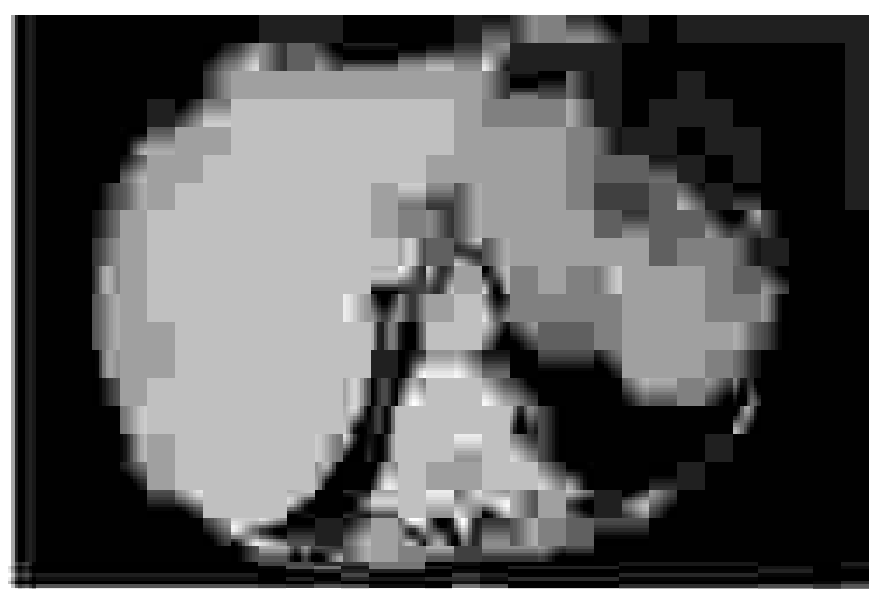

Fig. 1. En el TAC abdominal se observan varias imágenes hipodensas, algunas triangulares, en hígado y bazo, que corresponden a infartos isquémicos.

Estudio necrópsico. Se demostraron ganglios linfáticos con intensa deplección linfoide y una médula ósea con cambios postquimioterapia. Se advirtieron, además, hallazgos patológicos en los siguientes órganos. Pulmón: los alveolos estaban ocupados por hematíes y había focos de necrosis ampliamente distribuidos; existían también focos de necrosis con trombos arteriales formados por masas de hifas ramificadas, correspondientes a Aspergillus fumiga tus. Hígado: focos de necrosis coagulativa y hemorragias del parénquima hepático, de predominio periportal; en los espacios porta había trombos que estaban formados por fibrina, infiltrado inflamatorio y gran cantidad de hifas. Riñón: se apreciaron lesiones infárticas, con arterias obstruidas por trombos de fibrina, hifas e infiltrado de linfocitos. Bazo: se renocieron amplias áreas de necrosis coagulativa con arterias ocluidas por trombos, con hifas, fibrina e infitrado

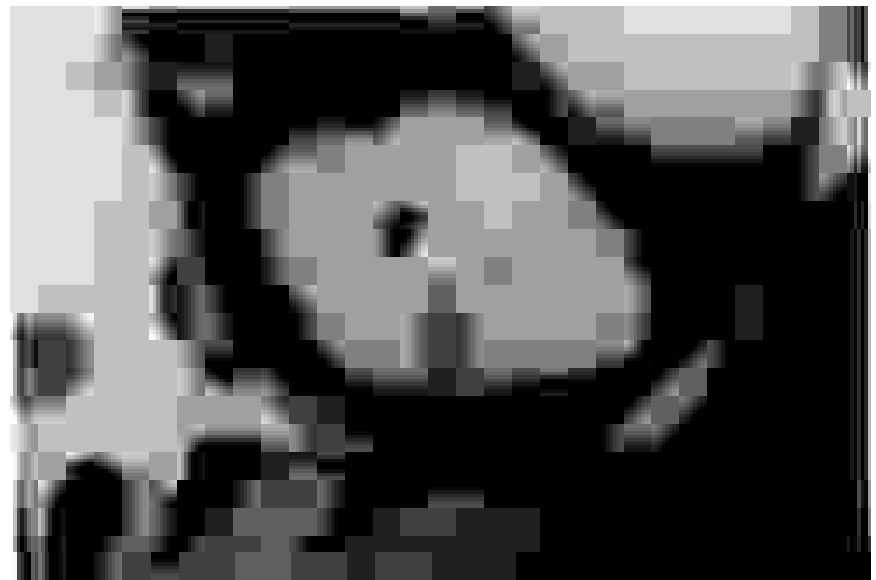

Fig. 2. En el riñón derecho se advierte una imágen triangular característica de un infarto isquémico.

celular. Cerebro: se observan áreas necrótico-hemorrágicas, con vasos trombosados por hifas en su interior, con fibrosis e infiltrado celular.

Caso 2: Varón de 65 años. Entre sus antecedentes destaca que hace dos años fue intervenido de cuádruple by-pass aortocoronario. Hace un año presentó un infarto cerebral isquémico de la cerebral media izquierda; se descartaron trombos cardiacos intracavitarios mediante ecocardiografía transtorácica. Poco después presentó embolia en arteria cubital derecha, que evolucionó satisfactoriamente. El motivo del último ingreso fue la presencia de fiebre, desorientación y aumento de la hemiparesia espática residual. A la exploración se objetiva paresia facial central con hemiplejía e hipertonía derecha y Babinsky derecho. Apertura espontánea de ojos. Obedece órdenes sencillas. Presenta cicatriz de esternotomía y no se percibían soplos, Glasgow 13. En el estudio analítico moderada anemia y leucocitosis de $34,7 \times 10^{3} / \mathrm{m \mu}(68 \% \mathrm{n} ; 27 \%$ 1; $5 \%$ e). Los parámetros bioquímicos convencionales eran normales salvo una creatinina de 2,8 mg. Los hemocutivos resultaron negativos. En la ecocardiografía, el ventrículo izquierdo no se apreció dilatado y la función ventricular global estaba conservada. Arteriografía cerebral: trombosis completa de la carótida interna izquierda. TAC craneal: lesión hipodensa en territorio de la arteria cerebral media izquierda compatible con infarto y efecto masa sobre ventrículo lateral. En los siguientes días se agravó el cuadro neurológico y falleció con fracaso multiorgánico.

Estudio necrópsico. Se encontró una aspergillosis parietal aórtica y una placa de ateroma en carótida primitiva izquierda colonizada por Aspergillus fumigatus. Asimismo se evidencó generalización de la enfermedad, con infartos isquémicos por probable embolización séptica en: cerebro, riñón, suprarrenales, páncreas, bazo, intestino y tiroides. En estas áreas había necrosis coagulativa, con arteriolas repletas de hifas ramificadas, fibrina e infiltrado celular inflamatorio.

\section{DISCUSIÓN}

La aspergilosis es una infección que complica a la inmunosupresión servera. Aparece, sobre todo, en pacientes con granulocitopenia, trasplantados y sometidos a tratamiento con corticoides o quimioterapia citiotóxica $(5,6)$. La infección es debida a una invasión masiva de los vasos sanguíneos por el hongo Aspergillus fumigatus (variedad más habitual). Característicamente los pulmones son el primer órgano invadido desde las vías aéreas superiores, donde llega por inhalación, lo que determina que clínicamente se exprese con un síndrome pulmonar (tos productiva, disnea y fiebre). La enfermedad 
diseminada ocurre en la mitad de los pacientes. La aortitis por Aspergillus se produce después de intervención de reemplazamiento aórtico o tras cirugía coronaria, sin que los pacientes tengan defecto inmunológico alguno, ni hayan sido sometidos a antibioterapia de larga duración, lo que induce a pensar que sea secundaria a una infección sobrevenida durante el acto quirúrgico $(7,8)$.

Nosotros damos a conocer dos casos de aspergilosis diseminada, el uno en un linfoma tratado con quimioterapia y el otro en un paciente no inmunodeprimido sometido a un cuádruple by-pass. Lo más notable es que en el linfoma se pudieron reconocer con el TAC cerebral infartos corticales y con el TAC toracoabdominal imágenes triangulares, hipodensas, sugestivas de infartos de riñón, hígado y bazo, que posteriormente fueron confirmadas en el estudio necróposico. En el otro paciente sólo se reconoció el infarto cerebral, mientras los infartos viscerales fueron un hallazgo de autopsia.

Hay algunos antecedentes en la literatura médica acerca de esta grave complicación tromboinfártica en la aspergilosis invasiva y en la aortitis aspergilar. Y así, en 1985, Ross (9) comunica el primer caso de infarto de miocardio por oclusión micótica del ostium de ambas coronarias, y al poco tiempo se conoció otro caso de infarto masivo de miocardio en el curso de una aspergilosis invasiva (10). También se ha descrito infarto esplénico total (11) e infartos renales (12), trombosis de los vasos hepáticos principales (arteria hepática y vena porta) (13), síndrome de Budd-Chiari (14), síndrome vena cava superior (15), y trombo flotante en la vena femoral (16). Hay varios casos publicados de infartos cerebrales asociados a la aspergilosis (17-19), que fueron confirmados en la autopsia y sospechados mediante TAC cerebral o RM; la RM mos- tró en uno de ellos irregularidades intraluminales en el sifón carotídeo derecho y en la arteria cerebral media proximal sugestivos de una arteritis (19).

No debe extrañar la complicación de infarto cerebral aparecida en la aspergilosis, ya que los accidentes cerebrovasculares isquémicos tienen múltiples causas desencadenantes, entre las que cabe considerar las infecciones (20), siendo entre ellas particularmente proclives a producir un infarto las que afectan directamente a las estructuras nerviosas, como las meningitis, donde se producen frecuentemente, al tiempo que actúan como marcador de gravedad. Recientemente Hashimoto encontró 8 casos de infarto cerebral en 43 casos de meningitis (21).

Pero la aspergilosis masiva se presenta a veces con una diátesis trombótica masiva, que puede comprometer a casi todos los órganos vitales, como se demostró palmariamente en nuestros dos pacientes. Y para comprenderla hay que buscar otras posibles vías patogénicas a esta trombosis. A la luz de nuestros hallazgos, pensamos que la ingente cantidad de hifas intravasculares, observadas en las áreas infartadas, pueden actuar a modo de émbolos sépticos con capacidad de obstrución de los vasos. Otra hipótesis alternativa es que la fibrina y la infiltración celular encontradas en la pared vascular denoten una arteritis por Aspergilus. Esta arteritis ha sido invocada por algunos autores, basándose incluso en modelos animales $(17,22)$.

Pensamos, en suma, que los infartos o multiinfartos viscerales costituyen una complicación de la aspergilosis invasiva que ha sido subestimada, como demuestran nuestros casos. Es necesario que aprendamos a reconocer estas formas emergentes de aspergilosis cada día más frecuentes en la era de la medicina tecnológica.

\section{Bibliografía}

1. Young RC, Bennet JE, Vogel CL. Aspergillosis: the spectrum of the diseases in 98 patients. Medicine 1970; 49: 147-173.

2. Fisher BD, SArmstrong D, Yu B, Irata T. Invasive aspergillosis: Progress in early diagnosis and treatment. Am J Med 1981; 71: 571577.

3. Gray R, Kaplan L, Matloff J, Uman S, Shachtmaan J. Aortic pseudoaneurysm with aspergillus aortitis. An unusual complication of coronary bypass surgery. Chest 1986; 89: 306-308.

4. Robbins JA, Mohanty PK, Mohanty LB. A aortic aspergilloma resulting in supravalvular aortic stenosis after aortocoronary by-pass surgery. Am Heart J 1983; 106: 163-166.

5. Ruutu P, Valtoneu V. An outbreak of invasive aspergillosis in a haematologig unit. Scand J Infect Dis 1987; 19: 347-351.

6. Hoffman G. Increased swareness of aspergillosis in acute leukemia patients. Haemat Blut Transf 1987; 30: 335-337.

7. Jenckes GA. Aspergillosis aortitis. J Thorac Cardiovasc Surg 1990: 99: 375-376.

8. Wandschneider W, Deutsch M. Fatal fungal infection of an ascending aortic graft. Thorax Cardiovasc Surg 1995; 432: 217-219.

9. Ross EM, Macher AB, Robets WC. Aspergillosis fumigatus thrombi causing total occlusion of both coronary arterial ostia, all four major epicardial coronary arteries and coronary sinus and associated with purulent pericarditis. Am J Cardiol 1985; 56: 499-500.

10. Laszewski M, Trigg M, Alarcon P, Giller R. Aspergillus coronary embolization causing acute myicardial infarction Bone Marrow Tranplant 1988; 3: 229-233.

11. Miller FH, Ma JJ, Total splenic infarction due to aspergillosis and AIDS. Clin Imaging 2000; 24: 362-363.

12. Landonio G, Nosari A, Gargantini L, cataldo F, Oreste P. Pulmonary and myocardial infaction secondary to arterial occlusion by Aspegillus

fumigatus in anll. Haematologica 1989; 74: 503-505.

13. Gavilan F, Torre-Cisneros J, Delgado M, Briceno J, Herrero C, Martinez L, et all. Thrombosis of the hepatic artery and portal vein secondary to invasive aspergillosis following liver trasplantation. Enferm Infecc Microbiol Clin 1998; 16: 127-129.

14. Young RC. The Budd-Chiari syndrome caused by Aspergillus; two patients with vascular invasion of the hepatic veins. Arch Intern Med 1969; 124: 754-757.

15. Gartenberg G, Einstein K, Jagirdar J. Superior vena cava syndrome caused by invasive aspergillosis. Chest 1978; 74. 671-672.

16. Noel AA, Gloviczki P, Charboneau JW. Free-floating femoral vein thrombus in a patient with aspergillosis. Int Angio 2000; 19: 75-78.

17. Matsumura S, Sato S, Fujiwara H, Takamatsu H, Kajiwara T, Yamashiro $\mathrm{K}$ et al. Cerebral aspergillosis as a cerebral vascular accident. No To Shinkey 1988; 40: 225-232.

18. Dayal Y, Weindling HK, Price DL. Cerebral infarction due to fungal embolus. A complication of aspergillosis infection on a aortic valve prosthesis. Neurology 1974; 24: 76-79.

19. Christophe C, Azzi N, Bouche B, Dan B, Levivier M, Ferster A. Magnetic resonance imaging and angiography in cerebral fungal vasculitis. Neuropediatrics 1999; 30: 218-220.

20. Rohuart F, Zagnoli F, Goas JY, Mocquard Y. Cerebral ischemic arterial accidents in yung adults. 40 cases. Rev Neurol 1993; 149: 547-553.

21. Hashimoto Y, Kimura K, Hirano T, Uchino M, Ando M. Cerebral infarction associated with meningitis. Rinsho Shinkeigaku 1996; 36: 1100-1103.

22. Tunew SS, Ehrahart EJ, Jensen HE, Joreman JH, Richter RA, Messic JB. Necrotizing mycotic vasculitis with cerebral infarction causes by Aspergillus nuger in a horse with acute typholocolitis. Vet Pathol 1999; 36: $347-351$. 